\title{
Inspection of Dissimilar Material Joints Using Ultrasonic Phased Arrays
}

\author{
Damira Smagulova ${ }^{1}$, Elena Jasiuniene ${ }^{1,2}$ \\ ${ }^{1}$ Prof. K. Barsauskas Ultrasound Research Institute, Kaunas University of Technology, \\ K. Barsausko St. 59, LT-51423 Kaunas, Lithuania \\ ${ }^{2}$ Department of Electronics Engineering, Kaunas University of Technology, \\ Studentu St. 50, LT-51367 Kaunas, Lithuania \\ damira.smagulova@ktu.edu
}

\begin{abstract}
Ultrasonic Inspection is widely used nondestructive testing technique. However, there are limited studies in the field of inspection of metal-composite adhesive joints. Since properties of dissimilar materials are different, reflection from the bonding between metal and composite occurs even without defects in the joint, what complicates the detection of defects in the joint. This work presents numerical and experimental study of ultrasonic inspection of metalcomposite adhesive joints. The numerical investigations were carried out using CIVA software. In this work phased array transducers of $3.5 \mathrm{MHz}$ and $5 \mathrm{MHz}$ frequency were studied and compared. It was determined, that there is a bigger possibility to detect delaminations using $3.5 \mathrm{MHz}$ transducer due to stronger signal, but the complexity of defect detection is caused by dissimilar joint itself - ultrasonic signal at the interface composite-metal is reflected even without defects in it. In order to improve the probability of defect detection in the interface the analysis of multiple reflections from the bonding zone was proposed.
\end{abstract}

Index Terms-Delamination; Dissimilar materials; Nondestructive testing; Phased arrays; Ultrasonic inspection.

\section{INTRODUCTION}

Adhesive bonding technology of dissimilar materials is widely used in different fields of industry due to its high efficiency and convenience. Metal-composite adhesive joints are used mainly to facilitate the weight and to obtain better characteristics of stiffness and strength of the structure itself. Usually joints of dissimilar materials are used in expensive constructions whose quality and uninterrupted operation is important not only in economic considerations but also in safety of human lives [1]-[4].

In adhesively bonded materials, different types of defects can appear which can cause a great harm. Thus, such structures must be checked on a regular basis and monitored in order to detect defects in advance, assess their damage and the possibility of further use. Defects such as voids, porosity, delaminations can occur at manufacturing stage because of a lack of adhesive or by the presence of foreign objects as well as during operation of the structure.

Manuscript received 10 April, 2018; accepted 2 August, 2018.

The research leading to these results has received funding from the European Union 7th Framework Programme under grant agreement $\mathrm{n}^{\circ}$ 310498, project SAFEJOINT "Enhancing structural efficiency through novel dissimilar material joining techniques".
Common problem of dissimilar material joint is poor joining that leads to appearance of delaminations [1], [2], [4]-[6].

Ultrasonic non-destructive inspection techniques are widely used for evaluation of quality of joints of different materials [3]-[7]. However, there are limited studies in the field of the inspection of metal-composite adhesive joints using ultrasonic techniques. The objective of this research was to develop ultrasonic technique, which would enable to find defects in metal-composite adhesive joints. An analysis of ultrasonic wave characteristics was proposed for evaluation of bonding quality of joint of dissimilar materials [8]-[10].

\section{Characteristics OF THE SAMPLE AND DEFECTS}

In order to select most suitable inspection technique the joint of dissimilar materials with known characteristics of artificial delaminations was analysed.

Sample under inspection was a joint of adhesively bonded Glass Fibre Reinforced Polymer (GFRP) and stainless steel There were three artificial delaminations between dissimilar layers in $300 \mathrm{~mm} \times 300 \mathrm{~mm}$ planar object. The full thickness of the object is $10.42 \mathrm{~mm}$, where steel thickness was $6.30 \mathrm{~mm}$ and GFRP $-4.12 \mathrm{~mm}$.

Artificial delaminations were made with polyethylene (PE) tape and a bit of oil that was spread over the layer to prevent the bonding between dissimilar layers. Delaminations have rectangular form and are located along the line in the centre of the object. Location of delaminations is shown in Fig. 1. Dimension of defects are given in Table $\mathrm{I}$.

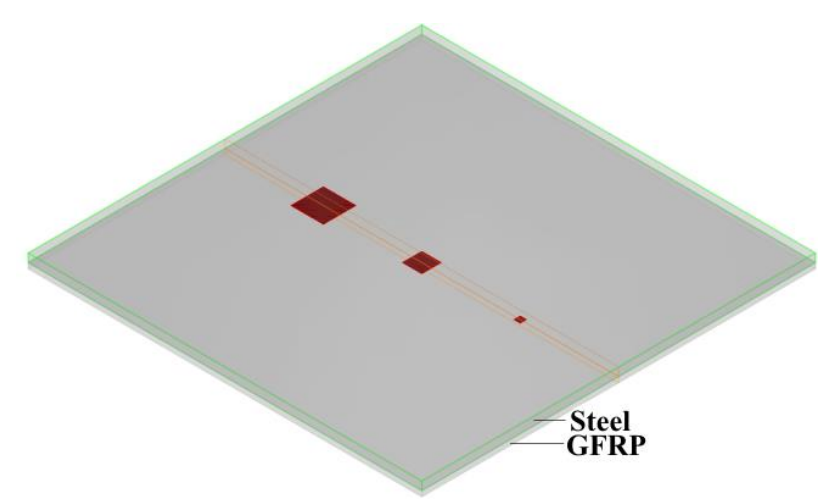

Fig. 1. Location of delaminations in joint of dissimilar materials. 
TABLE I. DIMENSION OF DEFECTS.

\begin{tabular}{|c|c|c|}
\hline Defects & Length, $\mathbf{~ m m}$ & Width, $\mathbf{~ m m}$ \\
\hline Delamination 1 & 25 & 25 \\
\hline Delamination 2 & 15 & 15 \\
\hline Delamination 3 & 5 & 5 \\
\hline
\end{tabular}

\section{SELECTION OF THE INSPECTION SET-UP}

Pulse Echo method is one of the most suitable ultrasonic technique that can be used for the inspection of the objects from one side if the access to other side is restricted. Defects of different orientation, type, location, size can be detected with longitudinal and shear waves as well as straight or angle beam technique [4], [6]. The object to be inspected has a simple planar geometry, thickness and depth of defects is large enough, orientation of defects is parallel to orientation of layers. Hence, as the most appropriate method for the inspection of adhesively bonded dissimilar materials pulse echo method was selected.

GFRP absorbs ultrasound waves more quickly and has a higher value of attenuation comparing to steel. Therefore, the value of amplitudes of reflected signal can be very low so it can be complicated to detect delaminations. Higher ultrasound velocity in steel layer was taken into account as well [5], [7]. The sample is inspected from metal side using one probe as emitter and receiver of ultrasound signal.

Acoustic impedances $z$ of steel and GFRP (1) are defined as a product of their densities $\rho$ and acoustic velocity $c$ [11]

$$
Z(R a y l)=\rho \times c
$$

Acoustic impedance plays a significant role in determining acoustic transmission and reflection from the boundary of the connection of two dissimilar materials having different acoustic impedances [11]. Acoustic impedances of GFRP and steel are shown in Table II.

TABLE II. ACOUSTIC IMPEDANCES OF DISSIMILAR MATERIALS.

\begin{tabular}{|c|c|}
\hline Material & Acoustic impedance $\boldsymbol{Z}$, MRayl \\
\hline Steel & 45.4 \\
\hline GFRP & 6.04 \\
\hline Oil & 0.74 \\
\hline Polyethylene & 1.76 \\
\hline Air & 0.000429 \\
\hline
\end{tabular}

Phased array transducers are selected to inspect object of adhesively bonded steel and GFRP. Phased array transducers consist of piezoelectric elements. Each element generates and transmits the signal. Characteristics of phased array transducers are shown in Table III.

TABLE III. CHARACTERISTICS OF PHASED ARRAY
\begin{tabular}{|c|c|c|}
\hline TRANSDUCERS. \\
\hline Transducer & $\mathbf{3 . 5} \mathbf{~ M H z}$ & $\mathbf{5 ~ M h z}$ \\
\hline Pattern & Linear phased array & Linear phased array \\
\hline Steering elements & 64 & 128 \\
\hline Elevation, mm & 7 & 7 \\
\hline Pitch, mm & 1 & 1 \\
\hline Virtual aperture & 8 & 8 \\
\hline Focus depth, mm & 6.30 & 6.30 \\
\hline
\end{tabular}

Hence, phased arrays cover a larger surface of the sample that excludes mechanical scanning along the defect length line. Focused signal was used for sample scanning and performed by altering time of the excitation pulses [12].

Frequencies were selected according to the thickness of steel, ultrasonic wavelength in steel, ultrasound velocity and attenuation. Ultrasonic wavelength depends on frequency and ultrasound velocity [8], [12], as in (2)

$$
\lambda=c / f
$$

where $\lambda$ is wavelength, $c$ - ultrasound velocity, $f$ - transducer frequency.

The parameters of the wavelength in steel are presented in

\begin{tabular}{|c|c|c|}
\hline Frequency, $\mathrm{MHz}$ & $\begin{array}{l}\text { Theoretical } \\
\text { velocity, } \mathrm{m} / \mathrm{s}\end{array}$ & Wavelength, mm \\
\hline 3.5 & \multirow{2}{*}{5940} & 1.7 \\
\hline 5 & & 1.2 \\
\hline
\end{tabular}
Table IV.

As a result, $3.5 \mathrm{MHz}$ and $5 \mathrm{MHz}$ were selected as the most appropriate frequencies for the sample inspection. Higher frequencies are not considered by reason of growth of attenuation.

\section{Modelling Results}

\section{A. CIVA Software and Experimental Set-up}

Frequency of $3.5 \mathrm{MHz}$ and $5 \mathrm{MHz}$ is selected for the inspection of characteristics of ultrasound in the object using CIVA software [13]. Using the CIVA software various models of inspection can be designed, results and parameters verified and analysed. The sample of dissimilar materials, delaminations as well as phased array transducers were designed in software. Inspection was performed from the metal side. The experimental set-up is shown in Fig. 2.

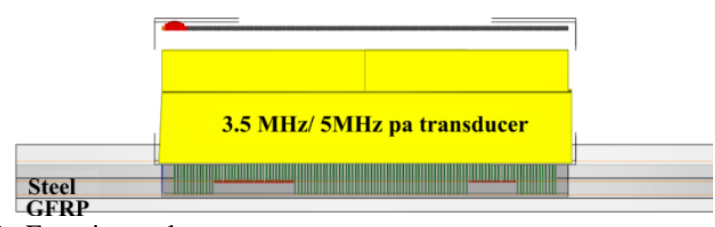

Fig. 2. Experimental set-up.

\section{B. Comparison of Amplitudes of Reflected Signals from Defected and Good Interface}

As a result of simulation in CIVA, 3 reflections were obtained. There are front reflection, reflection from delamination/good interface and reflection from the bottom of the sample. Comparison of amplitudes of ultrasonic waves reflected from good and defected areas of the sample using $3.5 \mathrm{MHz}$ and $5 \mathrm{MHz}$ phased array transducers are shown in Fig. 3 and Fig. 4.

Amplitude of reflection from the defect is $1 \mathrm{~dB}$ higher than reflection from the interface of the sample using 3.5 MHz phased array transducer. In the case of inspection, using $5 \mathrm{MHz}$ phased array transducer amplitude of ultrasound reflection from the defect is only $0.5 \mathrm{~dB}$ higher than reflection from the interface.

Since properties of dissimilar materials are different, the reflection from the bonding between steel and GFRP cannot be avoided. Therefore, delaminations can be hardly located only by comparing amplitude difference of reflections from good and defected area. As a result, using $3.5 \mathrm{MHz}$ transducer the possibility to detect delamination is a little higher comparing to $5 \mathrm{MHz}$ transducer but still complicated. 


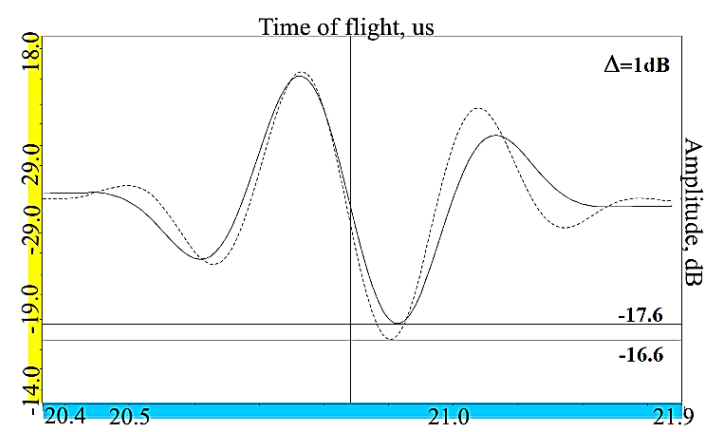

Fig. 3. A-scan of the inspection from the steel side using 3.5 $\mathrm{MHz}$ phased array transducer. Solid line of signal is the reflection from the interface, dotted line - the reflection from the defect.

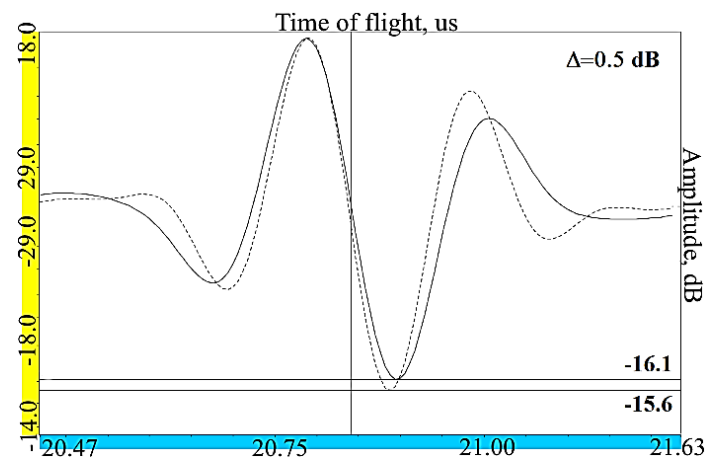

Fig. 4. A-scan of the inspection from the steel side using $5 \mathrm{MHz}$ phased array transducer. Solid line of signal is the reflection from the interface, dotted line - the reflection from the defect.

\section{Comparison of Signal Strength of $3.5 \mathrm{MHz}$ and $5 \mathrm{MHz}$ Transducers}

Difference of amplitude values of ultrasound reflected from the defect of the sample using $3.5 \mathrm{MHz}$ and $5 \mathrm{MHz}$ transducers is shown in Fig. 5.

Reflection from the defect using $3.5 \mathrm{MHz}$ transducer is $6.7 \mathrm{~dB}$ higher than reflection using $5 \mathrm{MHz}$ transducer. As a result, $5 \mathrm{MHz}$ transducer has less power comparing to $3.5 \mathrm{MHz}$ transducer. It is caused due to absorption of ultrasonic waves and attenuation rise by increasing frequency [8], [9]

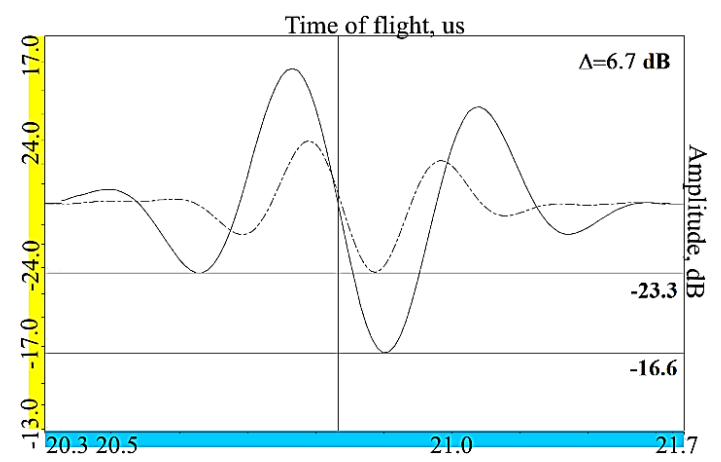

Fig. 5. Amplitudes comparison of the reflections from the defect. Solid line of signal is amplitude of $3.5 \mathrm{MHz}$ phased array, dotted line- amplitude of $5 \mathrm{MHz}$ phased array.

\section{Analysis of Multiple Ultrasonic Reflections}

In order to improve the probability of detection of the defects on the interface of the object the analysis of multiple reflections from interface zone was performed. Ultrasound reflections were calculated theoretically and compared to CIVA results [3], [4], [6], [14].

Time of flight of various ultrasonic wave paths in the sample were calculated theoretically [8], [12], as in (3)

$$
\tau=\frac{2 \times H_{\text {steel } / G F R P}}{c_{\text {steel } / G F R P}},
$$

where $\tau$ is the time of flight, $H$ - thickness of layers of dissimilar materials, $c$ - the ultrasound velocity in steel and GFRP.

Different wave paths of ultrasound in adhesively bonded GFRP and steel were studied. The time of flight of ultrasonic waves along different paths in the sample was calculated. Ultrasound propagation pathways were assigned to each reflected signal and shown in Fig. 6.

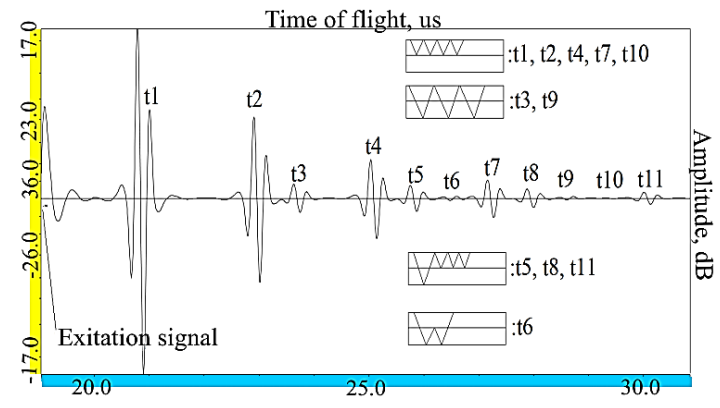

Fig. 6. Ultrasonic wave pathways.

A-scan comparison of reflections from the defect and reflections from the interface of the object is shown in Fig. 7.

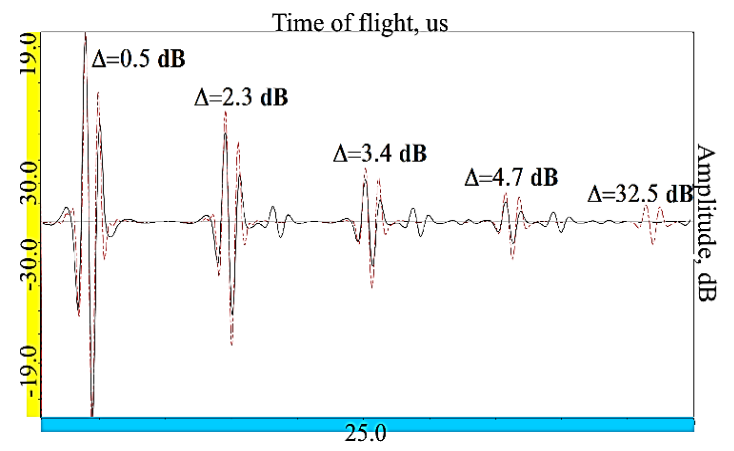

Fig. 7. A-scan comparison of reflection from the defect (dotted line) and from the interface (solid line).

The difference between the values of amplitude of reflected signals from the good and defected areas increases due to signal attenuation in steel and GFRP [8], [9]. Therefore, analysis of multiple reflections gives a higher possibility to locate defects in the joint of dissimilar materials.

\section{EXPERIMENTAL ULTRASONIC INSPECTION USING PULSE ECHO METHOD}

Omniscan measurement system was used for the defect inspection. All parameters of the transducer as frequency, aperture, steering elements of phased arrays, step were set. Characteristics of transducer were used same as in modelling part. Electronic scanning with one element step was performed. Special gel was used as a coupling media. Phased array transducer was mounted on the surface of the sample from metal side. The scanning was performed along the defect line in the center of the sample. The ruler was used as an auxiliary tool. Experimental set-up of the defect inspection from metal side using echo impulse mode is shown in Fig. 8. 


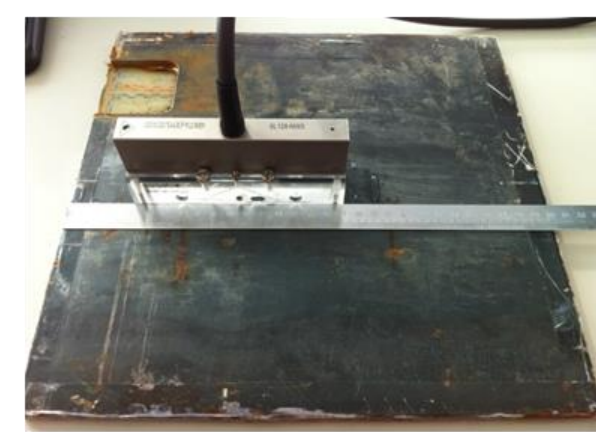

Fig. 8. Experimental set-up.

The inspection was performed to locate delaminations using $5 \mathrm{MHz}$ and $3.5 \mathrm{MHz}$ phased arrays. S-scan of the inspection of sample using $5 \mathrm{MHz}$ transducer is shown in Fig. 9 and S-scan of inspection of sample using $3.5 \mathrm{MHz}$ transducer is shown in Fig. 10.

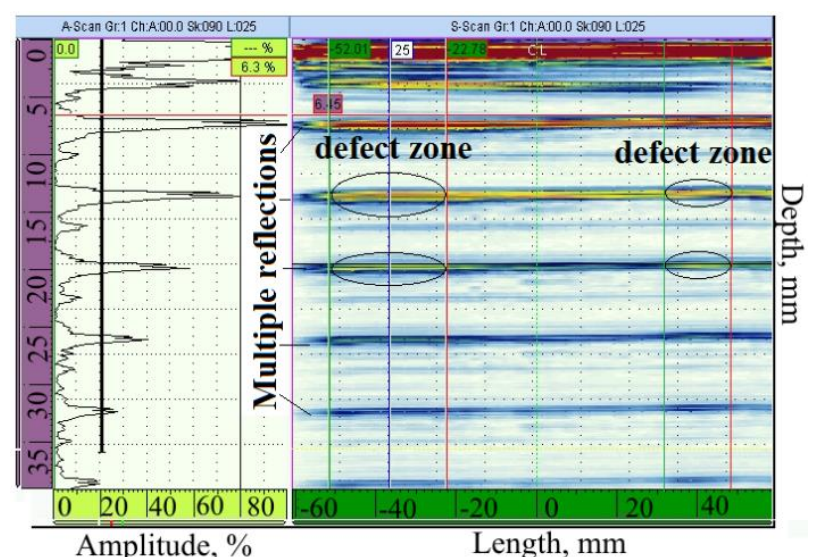

Fig. 9. S-scan of the inspection of first and second delamination in steel/GFRP sample using $5 \mathrm{MHz}$ phased array transducer.

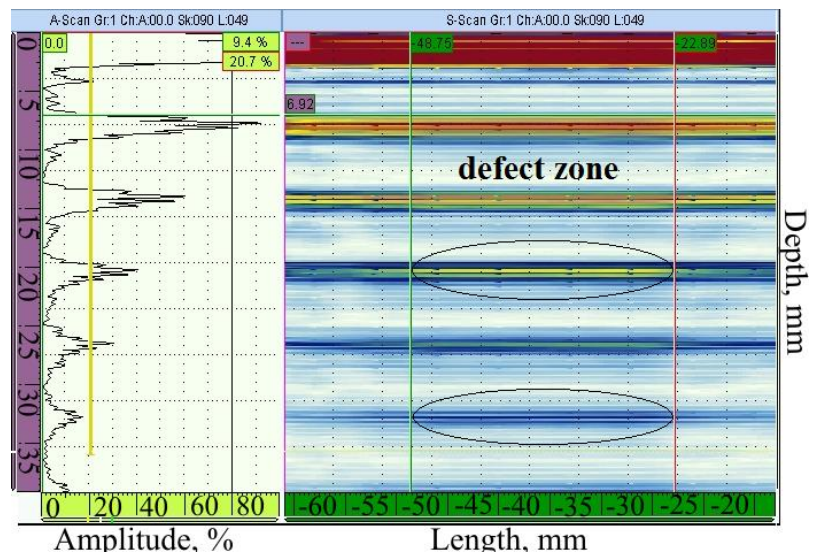

Fig. 10. S-scan of the inspection of first delamination in steel/GFRP sample using $3.5 \mathrm{MHz}$ phased array transducer.

According to S-scan, the depth of $6.30 \mathrm{~mm}$ of delaminations and dissimilar material bonding was defined.

In the case of inspections using $5 \mathrm{MHz}$ transducer the length of first delamination is $26 \mathrm{~mm}$, of second delamination is $15 \mathrm{~mm}$ and of third delamination is $20 \mathrm{~mm}$. In the case of inspection using $3.5 \mathrm{MHz}$ transducer the length of first delamination is $26 \mathrm{~mm}$, of second delamination is $15 \mathrm{~mm}$ and of third delamination is $20 \mathrm{~mm}$.

A number of errors can affect the measurements and final result of ultrasound inspection. The sources of errors are transducer frequency, wavelength of ultrasound, velocity, time of flight, focus depth, pitch of phased arrays, resolution of display of measurement system, and the operator himself [14].

\section{DISCUSSION}

Inspection of dissimilar metal-composite adhesive joint is a complex task, as the acoustic impedances of the adherents differ, meaning that there will be strong reflection at the interface of two dissimilar materials even without defect in it [3]-[5]. According to CIVA modelling results, it is hard to locate defects in both cases using $3.5 \mathrm{MHz}$ and $5 \mathrm{MHz}$ phased array transducers because of small amplitude difference of interface and defect reflections. It is caused by small differences of acoustic impedances of steel/GFRP and steel/PE and oil [11]. During experiment it was hard to detect amplitude difference between reflections from delamination and good interface. Hence, it proves the accuracy of results obtained during simulation.

In order to improve the probability of detection of the defects in the object the analysis of multiple reflections from the interface zone was performed [3], [4], [6]. In the subsequent reflected signals, the amplitude difference between good and defected areas increases, this gives us a greater possibility to detect delaminations. The same phenomenon was observed in experimental part where it was easier to detect defects from subsequent multiple reflections.

\section{CONCLUSIONS}

Investigation of adhesively bonded metal-composite using ultrasonic non-destructive testing was presented in this paper and the most suitable technique of detection of delaminations was developed. Delaminations are common problem in such joints but their detection is a complicated task due to great difference of properties of dissimilar materials. The field of metal-composite joints is not widely studied and there is a lack of investigations in this field.

Phased array transducers with a focused signal are defined as the best option for this investigation. The frequency of $3.5 \mathrm{MHz}$ is selected as more suitable with a greater probability of defect detection. The amplitude difference between reflection from delamination and not defected area is $1 \mathrm{~dB}$. Poor bond is easier to distinguish due to analysis of multiple reflections. The amplitude difference of reflections from deleminations and not defected interface reaches $32.5 \mathrm{~dB}$.

Further deeper investigation of joint of dissimilar materials is required to identify the best technique for detecting delaminations. Other computer software should be used for detailed analysis and various configurations of inspection should be observed with which the identification of such kind defects will be much easier.

\section{REFERENCES}

[1] J. Jahn, M. Weeber, J. Boehner, R. Steinhilper, "Assessmen strategies for composite-metal joining technologies - a review", Procedia CIRP, vol. 50, pp. 689-694, 2016. DOI: 10.1016/j.procir.2016.05.034.

[2] K. Martinsen, S. J. Hu, B. E. Carlson, "Joining of dissimilar materials”, CIRP Ann., vol. 64, no. 2, pp. 679-699, 2015. DOI: 10.1016/j.cirp.2015.05.006.

[3] D. Palumbo, R. Tamborrino, U. Galietti, P. Aversa, A. Tati, V. A. M. Luprano, "Ultrasonic analysis and lock-in thermography for 
debonding evaluation of composite adhesive joints", NDT E Int. vol. 78, pp. 1-9, 2016. DOI: 10.1016/j.ndteint.2015.09.001.

[4] M. A. Caminero, S. Pavlopoulou, M. Lopez-Pedrosa, B. G. Nicolaisson, C. Pinna, C. Soutis, "Analysis of adhesively bonded repairs in composites: Damage detection and prognosis", Compos. Struct., vol. 95, pp. 500-517, 2013. DOI: 10.1016/j.compstruct.2012.07.028.

[5] S. O. Olajide, "Progress on investigation on damage analysis in bonded polymer composites under fatigue", Int. J. Fatigue, vol. 96, pp. 224-236, 2017. DOI: 10.1016/j.ijfatigue.2016.12.016.

[6] A. Ganapathi, "Pulse echo ultrasonic testing of adhesive bonded joints for automotive applications", Ph.D. dissertation, Clemson University, 2010.

[7] M. E. Ibrahim, R. A. Smith, C. H. Wang, "Ultrasonic detection and sizing of compressed cracks in glass- and carbon-fibre reinforced plastic composites", NDT E Int., vol. 92, pp. 111-121, 2017. DOI: 10.1016/j.ndteint.2017.08.004.

[8] A. E. Powles, D. J. Martin, I. T. Wells, C. R. Goodwin, "Physics of ultrasound", Anaesth. Intensive Care Med., vol. 19, no. 4, pp. 202205, 2018. DOI: 10.1016/j.mpaic.2018.01.005.

[9] P. B. Wilkinson, D. A. Gunn, S. Holyoake, B. A. J. Dashwood,
C. R. Brett, J. G. Rees, "Low frequency acoustic and ultrasound waves to characterise layered media", NDT E Int., vol. 96, pp. 35-46, 2018. DOI: 10.1016/j.ndteint.2018.03.005.

[10] R. Tamborrino, D. Palumbo, U. Galietti, P. Aversa, S. Chiozzi, V. A. M. Luprano, "Assessment of the effect of defects on mechanical properties of adhesive bonded joints by using non destructive methods", Composites Part B: Engineering, vol. 91, pp. 337-345, 2016. DOI: 10.1016/j.compositesb.2016.01.059.

[11] The Impedance Measurement Handbook, A guide to measurement technology and techniques, 4th ed., Agilent Technologies Inc., USA, 2013, $140 \mathrm{p}$.

[12] D. Smagulova, E. Jasiuniene, "Ultrasonic non-destructive evaluation of dissimilar material joints", 12th student scientific conference on electronics, energy, telecommunications and automation (E2TA2015), Kaunas, Lithuania, 2015, pp. 8-12.

[13] CIVA 2015 User Manual (v11.1), Civa Extende, 2015.

[14] B. C. de Andrade Silva, M. S. Motta, J. E. F. de Oliveira, "Development of a methodology to determine thickness measurement uncertainties by ultrasonic test in aerospace parts", Materials Science Forum, vol. 758, pp. 89-97, 2013. DOI: 10.4028/www.scientific.net/MSF.758.89 